\title{
Intravitreal Injection for Diabetic Macular Edema as Adjunctive Therapy for Proliferative Diabetic Retinopathy: A Retrospective Study
}

\author{
Wissam Aljundi (iD)', Shady Suffo', Cristian Munteanu', Achim Langenbucher², Berthold Seitz (D)', \\ Alaa Din Abdin' \\ 'Department of Ophthalmology, Saarland University Medical Center UKS, Homburg, Saar, Germany; ${ }^{2}$ Institute of Experimental Ophthalmology, \\ Saarland University, Homburg, Saar, Germany \\ Correspondence: Wissam Aljundi, Tel +49684 I I622387, Fax +49684 I I622400, Email wissam.aljundi@uks.eu
}

Purpose: To detect the impact of intravitreal injection (IVI) therapy with sole anti-vascular-endothelial-growth-factor (VEGF) or combined with steroids treating diabetic macular edema (DME) on activity of proliferative diabetic retinopathy (PDR) based on total number of panretinal photocoagulation (PRP) spots needed within 2 years.

Patients and Methods: A retrospective study of 102 eyes with primary-onset PDR and minimum follow-up of 24 months divided into 2 groups: Group 1 (G1) 40 eyes received only PRP and did not develop DME. Group 2 (G2) 62 eyes received additional IVItherapy due to concomitant DME, with anti-VEGF only (subgroup 2a, G2a) or in combination with steroids (subgroup 2b, G2b). Main outcomes: central macular thickness (CMT, $\mu \mathrm{m})$, best-corrected visual acuity (BCVA, LogMAR) and total number of needed PRP spots and IVI after 24 months.

Results: CMT was significantly higher in G2 compared to G1, initially $(p<0.01)$ and after 24 months $(p=0.01)$. CMT was significantly higher in G2b compared to G2a, both initially $(\mathrm{p}=0.01)$ and after 24 months $(\mathrm{p}<0.01)$. BCVA was significantly higher in G1 compared to G2, initially and after 24 months $(p=0.01)$. BCVA was not significantly different between the two subgroups, initially $(p=0.54)$ and after 24 months $(p=0.29)$. The total number of PRP spots was significantly higher in G1 compared to G2 ( $<<0.01)$ but not significantly different between the subgroups $(\mathrm{p}=0.8)$.

Conclusion: Regardless of severity of concomitant DME, IVI with sole anti-VEGF or combined with steroids reduced the total number of PRP spots needed within 2 years significantly indicating a favorable effect on activity of PDR.

Keywords: proliferative diabetic retinopathy, diabetic macular edema, intravitreal injection, panretinal photocoagulation, anti-VEGF, steroids

\section{Introduction}

Diabetic retinopathy is the most common complication of diabetes, which advances from non-proliferative- (NPDR) to proliferative diabetic retinopathy (PDR). Diabetic macular edema (DME) is a complication independent from DR and occurs in approximately $6.8 \%$ of DM patients. PDR and the DME are the leading causes of vision loss in patients with DM. ${ }^{1-4}$

Several clinical and preclinical studies indicate that the increased vascular endothelial growth factor (VEGF) is a key mediator in the development of retinal neovascularization (NV) in PDR. ${ }^{5-7}$ Moreover, many features of inflammation were pathologically elevated in PDR and DME. ${ }^{8-12}$

The panretinal photocoagulation (PRP) is still the method of choice for the treatment of PDR. It leads to a reduction of the VEGF production and the oxygen need and thus reduced the activity of PDR. However, PRP could lead to worsening of the preexisting DME. The Diabetic Retinopathy Clinical Research Network (DRCR.net) Protocol S has shown that intravitreal injection of ranibizumab is also an effective method to reduce the activity of PDR and non-inferior to PRP. ${ }^{13}$ 
Clinicians nowadays are lost somewhat in the optimal treatment of PDR with or without DME: the old trend is to do PRP and the new school do injections. In this study, we tried to evaluate a possible midway solution, ie, to ascertain whether a combination could lead to less laser spots and hence less damage or less destruction of the retina.

\section{Materials and Methods}

\section{Patients and Materials}

Retrospectively, we analyzed the data of all patients with primary onset PDR, who presented at our department between 01.01.2016 and 31.12.2018, and included 102 eyes who met our below-mentioned inclusion/exclusion criteria. The eyes were divided into 2 main groups according to the presence of a concomitant DME at baseline. The observation period started at the diagnosis of PDR and ended after 24 months. DME was defined as the presence of any retinal thickening or hard exudates at the posterior pole.

- Group 1 (G1: eyes without DME, treated only with PRP).

- Group 2 (G2: eyes with concomitant DME at baseline, treated with PRP and IVI).

PDR was classified according to The Early Treatment Diabetic Retinopathy Study Research Group (ETDRS) classification using findings of fundus- and fluorescein angiography (FAG) into 2 forms: early- and high-risk PDR. High-risk characteristics are defined as the following: neovascularization of the optic disc (NVD) greater than 1/3 to $1 / 4$ of the optic disc diameter, NVD less than $1 / 3$ to $1 / 4$ of the optic disc diameter with vitreous or preretinal hemorrhage, neovascularization elsewhere (NVE) with vitreous or preretinal hemorrhage. If none of the above mentioned criteria are present, the PDR is defined as an early PDR. The PDR is defined as advanced in case of a retinal detachment, persistent vitreous hemorrhage or NVG. ${ }^{14}$ Furthermore, PDR was classified as active when NV was detected in FAG and inactive when no signs of NV were detected. ${ }^{15}$

Patients in G2 were further divided into 2 subgroups according to the applied IVI agent:

- Subgroup 2a (G2a: eyes treated with sole IVI therapy with anti-VEGF agents during the observation period).

- Subgroup 2b (G2b: eyes treated initially with anti-VEGF agents and then switched to steroids).

\section{The Inclusion Criteria}

- Participants were of both genders, aged 18 years or above and had either insulin-dependent diabetes mellitus (IDDM) or non-insulin-dependent diabetes mellitus (NIDDM).

- Primary onset PDR.

- Minimum follow-up of 24 months.

\section{The Exclusion Criteria}

- History of DR treatments, including PRP or any previous intravitreal injection.

- Eyes with concomitant retinal pathologies leading to cystoid macular edema, increased retinal thickness or retinal neovascularization (eg, eyes with vascular occlusion, history of uveitis or postoperative macular edema, age-related macular degeneration) were excluded.

- Eyes in G1 which had developed a DME during the observation period.

- Advanced PDR at baseline.

- Patients with vitreomacular traction (VMT) or proliferative vitreoretinopathy (PVR) in macula area.

- Pregnancy.

\section{Primary Outcome Measures}

- The central macular thickness (CMT, in $\mu \mathrm{m}$, as measured by Spectral Domain Optical Coherence Tomography (Spectralis SD-OCT; Heidelberg Engineering, Heidelberg, Germany)). 
- The best-corrected visual acuity (BCVA, initially recorded in decimal and converted to LogMAR before performing the statistics).

- The total number of the applied PRP spots at the end of the observation period.

\section{Treatment}

PRP:

The PRP was done in all eyes of both groups using a conventional argon laser (wave-length $532 \mathrm{~nm}$, VISULAS, 532 s, Carl Zeiss Meditec AG, Jena, Germany; laser settings: pulse energy: 150-400 mW, pulse duration $150 \mathrm{ms,} \mathrm{spot} \mathrm{size}$ $400 \mu \mathrm{m}$ ) and a Goldman-SuperQuad 160 contact lens (double magnification) around arcade towards equator.

IVI:

- All injections were carried out in a designated intravitreal injection center in our Department of Ophthalmology at the Saarland University Medical Center. ${ }^{16}$

- The initial IVI therapy in all eyes of group 2 was with anti-VEGF [Bevacizumab (Avastin 1.25mg/0.05mL; Roche, Grenzach, Germany), Ranibizumab (Lucentis $0.5 \mathrm{mg} / 0.05 \mathrm{~mL}$; Novartis, Nuremberg, Germany) and Aflibercept (Eylea $2 \mathrm{mg} / 0.05 \mathrm{~mL}$; Bayer Healthcare Pharmaceuticals, Berlin, Germany)] and was applied according to the regime described below.

- Patients in G2b were switched to steroids [Dexamethasone (Ozurdex 0.7mg; Allergan, Inc., Frankfurt, Germany) or

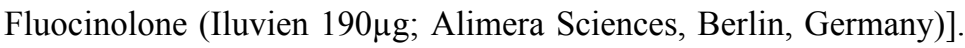

The IVI-Regime used in all eyes of both subgroups:

(I) Upload phase with 4x IVI with anti-VEGF agents in monthly intervals.

(II) the further treatment took place after the upload phase as long as:

- none of the following discontinuation criteria were present:

- BCVA (in LogMAR) $>1.3$ (except in case of media opacity) without prospect of improvement.

- No further improvement was morphologically suggested (atrophy, ischemia).

In this case therapy was discontinued or the drug was switched.

- none of the following success criteria were present:

- BCVA (in LogMAR) $<0$.

- Foveal edema was no longer present.

In this case the therapy was paused.

- one of the efficacy criteria was still fulfilled:

- Further improvement of visual acuity ( $>5$ letters in the last 3 months).

- Decrease of edema ( $>10 \%$ in the last 3 months).

(I) Re-treatment in case of renewed morphological or functional reactivity:

- With 2x upload (2-24 months after last injection).

- With $4 x$ upload (>24 months after last injection).

\section{The Statistical Analysis}

Data were collected with Microsoft Excel 2010 (Microsoft Corporation, Redmond, WA) and analyzed with SPSS version 27 (SPSS Inc, Chicago, IL). 
We used Mann-Whitney $U$-Test for not normally distributed variables and $t$-test for normally distributed, and to compare frequencies we used the Chi-square $\left(\chi^{2}\right)$ test. $\mathrm{P}$ values $<0.05$ were considered statistically significant.

\section{Main Limitations of This Study}

The main potential limitations of our study were the retrospective nature of the work, a relatively small population from a single medical center, and the use of decimal visual acuity as opposed to ETDRS vision charts. However, to obtain more reliable values to describe BCVA improvement, decimal values were converted to LogMAR equivalents before running the statistics.

In this study, the Fluorescence angiography was performed with the Heidelberg Retina Angiograph (HRA 2), which does not provide ultra-widefield images, which could make the accurate calculation of the total retinal area. Thus, the ischemia index would have been technically inaccurate and was not included in the present study.

\section{Results}

\section{Baseline Characteristics (Tables I and 2)}

The Central Macular Thickness (CMT, in $\mu \mathrm{m})$ : (Figure IA and B)

The CMT (in $\mu \mathrm{m}$ ) at the first visit was $296.6 \pm 69.5$ in G1 and 432.1 \pm 164.2 in G2 (p<0.01). This was $308.9 \pm 59.7$ in G1 and $382.1 \pm 143.8$ in $\mathrm{G} 2$ at the end of the observation period $(\mathrm{p}=0.01)$.

The CMT in G2a was significantly lower at the first and the last visit than in G2b: at the first visit: $385.1 \pm 128.9$ vs $474.8 \pm 182.2(\mathrm{p}=0.01)$, at the end of the observation period: $338.8 \pm 132.3$ vs $425.0 \pm 144.0(\mathrm{p}<0.01)$ in G2a vs G2b.

\section{The Best-Corrected Visual Acuity (BCVA, in LogMAR): (Figure 2A and B)}

The initial BCVA was $0.27 \pm 0.44$ in $\mathrm{G} 1$ and $0.36 \pm 0.39$ in $\mathrm{G} 2(\mathrm{p}=0.01)$. At the end of the observation period, the BCVA was $0.28 \pm 0.31$ in $\mathrm{G} 1$ and $0.46 \pm 0.45$ in $\mathrm{G} 2(\mathrm{p}=0.01)$.

The initial BCVA was $0.31 \pm 0.40$ in G2a and $0.41 \pm 0.37$ in $\mathrm{G} 2 \mathrm{~b}(\mathrm{p}=0.54)$. At the end of the observation period, the BCVA was $0.42 \pm 0.44$ in $\mathrm{G} 2 \mathrm{a}$ and $0.5 \pm 0.46$ in $\mathrm{G} 2 \mathrm{~b}(\mathrm{p}=0.29)$.

\section{The Number of the Applied PRP Spots}

The total number of applied PRP spots at the end of the observation period was $2427.4 \pm 885.0$ in G1 and 1528.8 \pm 468.6 in $\mathrm{G} 2(\mathrm{p}<0.01)$. This was $1428 \pm 424.6$ in G2a and $1552.8 \pm 512$ in $\mathrm{G} 2 \mathrm{~b}(\mathrm{p}=0.6)$.

Table I Baseline Characteristics of the Two Main Groups

\begin{tabular}{|l|l|l|l|}
\hline \multicolumn{4}{|c|}{ Baseline Characteristics in the Main Groups (Means \pm SD) } \\
\hline Variable & GI (n = 40) & G2 (n= 62) & p-value \\
\hline Patient age (years) at baseline & $60 \pm 17$ & $61 \pm 14$ & 0.98 \\
\hline Gender (male/female) & $24 / 16$ & $41 / 21$ & 0.52 \\
\hline Type of DM (type I/type 2) & $22 / 18$ & $32 / 30$ & 0.84 \\
\hline HbAlc at baseline & $8.25 \pm 0.74 \%$ & $8.44 \pm 0.86 \%$ & 0.23 \\
\hline Duration of DM (years) at baseline & $26.16 \pm 12.61$ & $20.29 \pm I I .82$ & $0.025 *$ \\
\hline Type of PDR (early/high-risk/advanced) & $33 / 7 / 0$ & $50 / 12 / 0$ & 0.81 \\
\hline
\end{tabular}

Notes: *Significant difference between the two groups using the Mann-Whitney U-test: $\mathrm{P}<0.05$; Group I (GI) eyes with only proliferative diabetic retinopathy (PDR), treated only with panretinal photocoagulation (PRP). Group 2 (G2) eyes with PDR and concomitant diabetic macular edema (DME), treated with PRP and intravitreal injection (IVI) therapy. Results are listed with mean \pm standard deviation; the $\mathrm{p}$ values refer to statistical differences between two groups.

Abbreviations: DM, Diabetes mellitus; Type I, insulin dependent diabetes mellitus; Type 2, non-insulin dependent diabetes mellitus; HbAIc, glycated hemoglobin; PDR, proliferative diabetic retinopathy. 
Table 2 Baseline Characteristics of the Two Subgroups (Eyes with Proliferative Diabetic Retinopathy (PDR) and Concomitant Diabetic Macular Edema (DME), Treated with Panretinal Photocoagulation (PRP) and Intravitreal Injection (IVI) Therapy)

\begin{tabular}{|l|l|l|l|}
\hline \multicolumn{4}{|c|}{ Baseline Characteristics in the Subgroups (Means \pm SD) } \\
\hline Variable & G2a $(\mathbf{n}=\mathbf{3 0})$ & G2b (n= 32) & p-value \\
\hline Patient age (years) at baseline & $58 \pm 13$ & $64 \pm 13$ & 0.07 \\
\hline Gender (male/female) & $21 / 9$ & $20 / 12$ & 0.53 \\
\hline Type of DM (type I/type 2) & $15 / 15$ & $17 / 15$ & 0.80 \\
\hline HbAlc at baseline & $8.62 \pm 0.79 \%$ & $8.28 \pm 0.91$ & 0.12 \\
\hline Duration of DM (years) at baseline & $17.93 \pm 10.07$ & $22.50 \pm 13.02$ & 0.21 \\
\hline Type of PDR (early/high-risk/advanced) & $26 / 4 / 0$ & $24 / 8 / 0$ & 0.245 \\
\hline
\end{tabular}

Notes: Group 2a (G2a) eyes treated with PRP and a sole IVI therapy with anti-VEGF agents during the observation period. Group 2b (G2b) eyes treated with PRP and IVI initially with anti-VEGF agents and then switched to steroids. Results are listed with mean \pm standard deviation; the $p$ values refer to statistical differences between two groups.

Abbreviations: DM, Diabetes mellitus; Type I, insulin dependent diabetes mellitus; Type 2, non-insulin dependent diabetes mellitus; HbAIc, glycated hemoglobin; PDR, proliferative diabetic retinopathy.

\section{The Number of IVI Injections}

A detailed description of the performed IVI in G2 and both subgroups is shown in Figure 3. The total number of IVI performed in G2 at the end of the observation period was $11 \pm 4$. In detail, this was $10 \pm 4$ in G2a (all of them were with anti-VEGF agents) and $12 \pm 4$ in G2b ( $8 \pm 4$ of which with anti-VEGF agents and $4 \pm 2$ with steroids) $(\mathrm{p}=0.035)$. Switching to steroids occurred in G2b after a mean of $9.9 \pm 4.7$ months.

\section{Discussion}

Several previous studies reported a positive impact of IVI therapy with anti-VEGF agents on PDR in terms of slowing down the progression and severity of PDR. ${ }^{17-19}$ According to the PRIDE study, Ranibizumab monotherapy was more effective than PRP in reducing activity of PDR in absence of DME between baseline and Month 12 in a total of 106 patients. ${ }^{17}$ Similarly, the CLARITY study reported a better visual acuity gain outcome at 1 year in patients with PDR treated with intravitreal Aflibercept than in patients treated with PRP in the absence of DME too. ${ }^{18}$

A

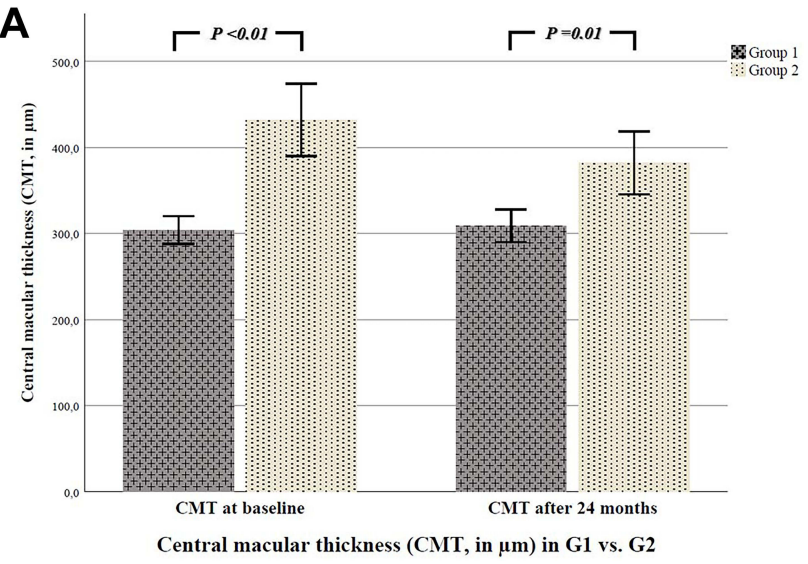

B

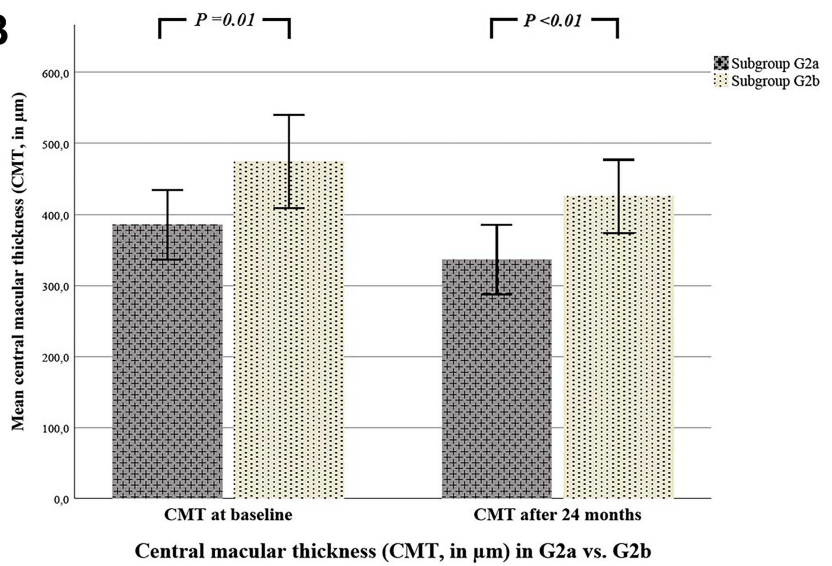

Figure I (A) There was a statistically significant difference regarding CMT $(\mu \mathrm{m})$ between both main groups at baseline $(\mathrm{P}<0.0 \mathrm{I})$ and after 24 months $(\mathrm{P}=0.0 \mathrm{I})$. Group I (GI) eyes with only proliferative diabetic retinopathy (PDR), treated only with panretinal photocoagulation (PRP). Group 2 (G2) eyes with PDR and concomitant diabetic macular edema (DME), treated with PRP and intravitreal injection (IVI) therapy. The $p$ values refer to statistical differences between two groups. (B) There was a statistically significant difference regarding CMT $(\mu \mathrm{m})$ between both subgroups at baseline $(p=0.0 \mathrm{I})$ and after 24 months $(p<0.0 \mathrm{I})$. Group 2a $(G 2 \mathrm{a})$ eyes treated only with PRP and a sole IVI therapy with anti-VEGF agents during the observation period. Group $2 b(\mathrm{G} 2 \mathrm{~b})$ eyes treated with PRP and IVI therapy initially with anti-VEGF agents and then switched to steroids. The $p$ values refer to statistical differences between two groups. 


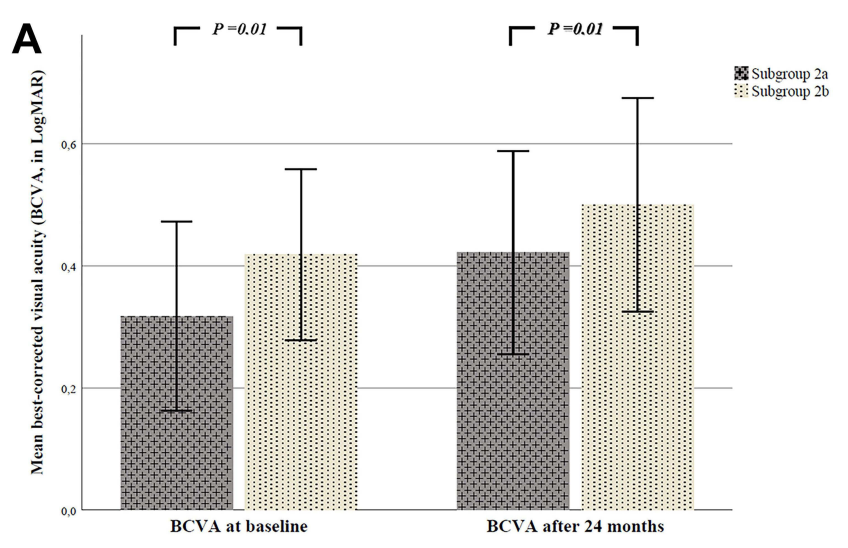

Best-corrected visual acuity (BCVA, in LogMAR) in G2a vs. G2b

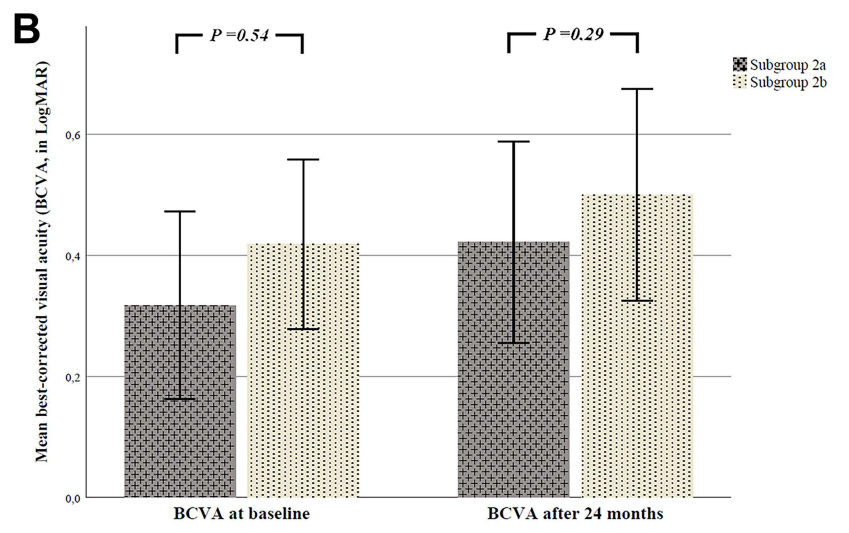

Best-corrected visual acuity (BCVA, in LogMAR) in G2a vs. G2b

Figure 2 (A) There was a statistically significant difference regarding BCVA (LogMAR) between both main groups at baseline and after 24 months ( $\mathrm{P}=0.0 \mathrm{I})$. Group I (GI) eyes with only PDR, treated only with PRP. Group 2 (G2) eyes with PDR and concomitant DME, treated with PRP and IVI therapy. The $P$ values refer to statistical differences between two groups. (B) There was no statistically significant difference regarding BCVA (LogMAR) between both subgroups at baseline $(p=0.54)$ and after 24 months $(p=$ 0.29). Group 2a (G2a) eyes treated only with PRP and a sole IVI therapy with anti-VEGF agents during the observation period. Group $2 b$ (G2b) eyes treated with PRP and IVI therapy initially with anti-VEGF agents and then switched to steroids. The $p$ values refer to statistical differences between two groups.

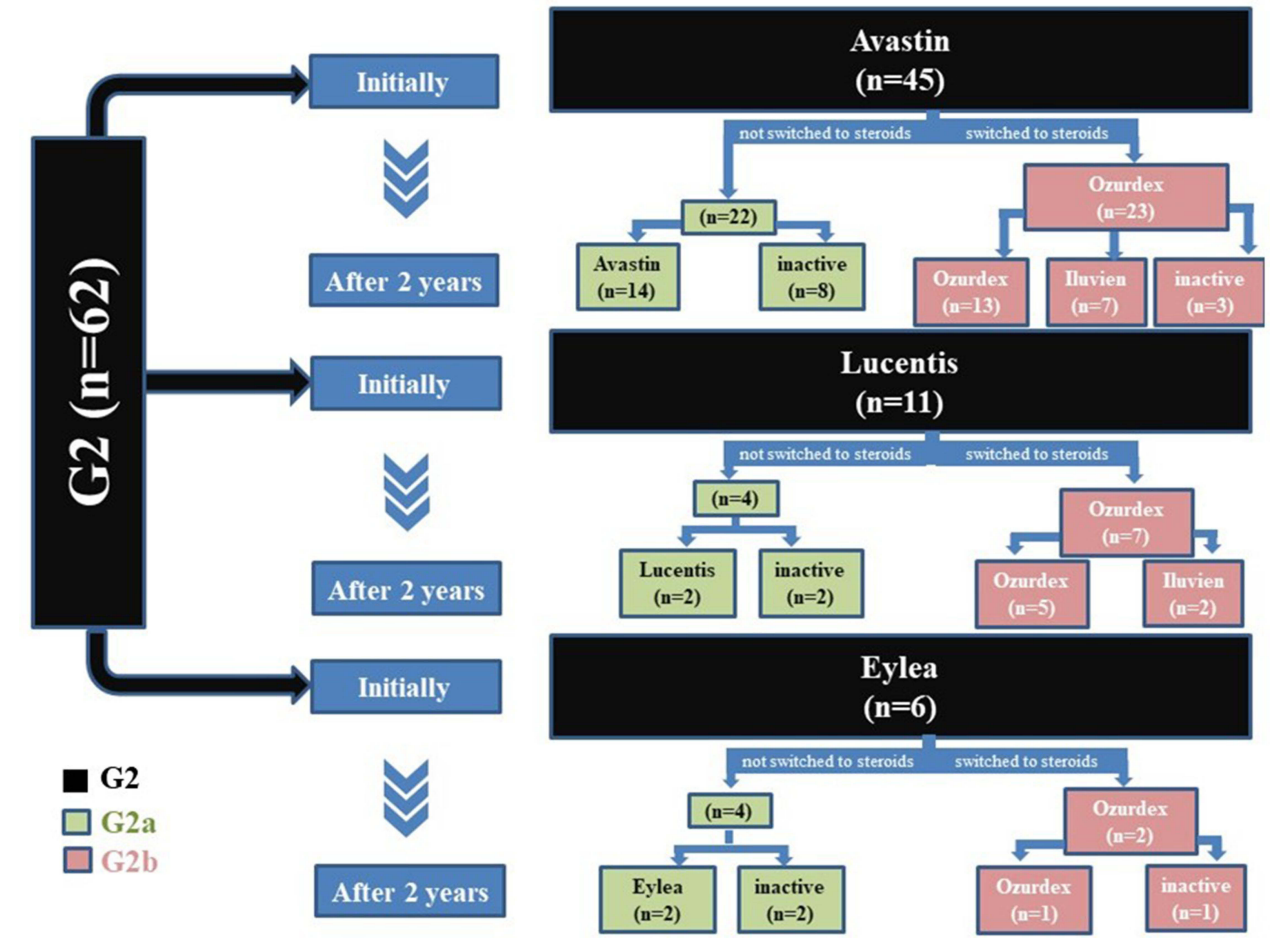

Figure 3 A detailed description of the intravitreal therapy in both subgroups within the observation period (24 months). Group 2a (G2a) eyes treated only with PRP and a sole IVI therapy with anti-VEGF agents during the observation period. Group $2 b(G 2 b)$ eyes treated with PRP and IVI therapy initially with anti-VEGF agents and then switched to steroids.

Unlike previously published studies, the present study examined the impact of a sole IVI therapy with anti-VEGF agents, as well as, in combination with steroids, used to treat DME on the activity of early- and high-risk PDR.

We found that the combined treatment in G2 with IVI and PRP therapy has a better outcome on the activity of PDR compared to monotherapy with PRP in terms of a significantly lower number of needed PRP therapy after 24 months. Furthermore, switching to steroids in G2b did not lead to a higher number of PRP spots required compared to eyes in G2a, which received a sole IVI therapy with anti-VEGF agents alone. This may suggest that IVI therapy with steroids could be a possible alternative to reduce the activity of PDR, too. 
The baseline characteristics were comparable in all groups and subgroups. There were no significant differences regarding age and gender of the patients, also regarding DM type and glycated hemoglobin (HbAlc) at the first visit. Since DME is a DM complication independent of DR, it was not surprising that DM duration differed between the two main groups, which however did not differ significantly between the two subgroups with DME. Moreover, a similar nonsignificantly different PDR level was present in all patients of the two main and subgroups, so that we could compare the development of PDR between the two main and subgroups afterwards.

Our main purpose was to investigate the impact of IVI therapy performed in both subgroups on the needed PRP therapy and consequently on the activity of PDR at the end of the 2 years. In this context, we found that the number of PRP spots used at the end of the observation period was significantly higher in G1 compared to G2, which supports the previous published studies concerning the positive effect of IVI therapy agents on the activity of PDR. ${ }^{17-19}$ Furthermore, we found no significant difference in the total number of PRP spots at the end of the observation period between the two subgroups, which suggests that switching to steroids may also have a non-inferior positive effect on the activity of PDR compared to a sole IVI therapy with anti-VEGF agents.

The anti-inflammatory role of steroids has been studied widely for a long time. It is well known that steroids prevent vasodilatation and increased vascular permeability, which occurs following an inflammatory event. They also reduce leukocyte migration into inflamed areas, leading to a new protein synthesis. ${ }^{20-22}$

It is a fact that inflammation plays a major role in the development of both PDR and DME. In patients with PDR, increased vitreous concentrations of various inflammatory cytokines, neurotrophins and many other inflammatory mediators along with other growth factors such as VEGF have been reported. Angiogenic response of endothelial cells is regulated by these inflammatory mediators and growth factors as well, which leads to the development of new vessels in PDR. The importance of inflammation in this context has been supported by the fact that blocking inflammation reduces neovascularization. ${ }^{23}$

These pathophysiological facts explain the long-standing use of steroids to treat DME and support our assumption that steroids may play a further role in the treatment of PDR.

DME in G2b seemed to be more aggressive and severe than in G2a, which led to the significantly higher CMT both initially and at the end of the 2 years follow up. Nevertheless, the number of used PRP spots was not significantly different between the two subgroups at the end of the observation period, which implies that IVI therapy with sole antiVEGF agents or in combination with steroids could reduce the number of used PRP spots regardless of the severity of DME.

This presumption that the IVI therapy performed due to DME had led to the reduction of used PRP spots, and consequently to significantly fewer PRP procedures could be supported by our recent internal statistics, since we had about $50 \%$ less number of PRP procedures and in contrast about $83 \%$ more IVIs in our department in the last four years. ${ }^{16}$

On the other hand, and due to the existing DME, CMT was significantly higher in G2 compared to G1 at baseline and at the end of the 2 years interval.

In contrast to previously published papers, such as the DRCR.net Protocol S study, ${ }^{13}$ BCVA did not represent an important parameter in our study due to the presence of the DME in G2. The DRCR.net Protocol S showed that the visual outcome was not worse under IVI therapy with ranibizumab compared to a monotherapy with PRP for PDR patients in absence of DME. As expected, BCVA was in our study significantly higher in G1 than in G2 both initially and at the end of the 2 years interval due to the presence of the DME.

Our findings were supported by a recent 2020 paper that compared daily rates of PRP with those of IVI therapy in 3656 eyes with early-onset PDR between 2012 and 2019. This paper showed that the rate of PRP for the treatment of PDR decreased significantly after the publication of Protocol S, while the rate of IVI with anti-VEGF increased significantly. ${ }^{24}$

However, it should be considered that the injection temporarily reduces VEGF drive and minimal laser treatment does not necessarily lead to well-controlled PDR in the long term with the risk of persistent NV activity and recurrence of vitreous hemorrhage. This is especially true for patients who skip FAG follow-up. Thus, the finding that fewer lasers are needed does not mean that PDR can be better controlled in the long term, but the contrary may be true. 
Based on the non-significant difference between anti-VEGF alone or combination with steroid therapy in reducing PDR activity in our subgroups, and the current evidence of the inflammatory component of PDR, the question arises whether steroid therapy may play a role in the treatment of PDR in the future. Here, future studies are of great importance.

In summary, we report that regardless of severity of concomitant DME, IVI with sole anti-VEGF or after switching to steroids led to significant reduction of supplemental PRP spots and indicating a favorable effect on the activity of PDR.

\section{Abbreviations}

IVI, Intravitreal Injection; VEGF, Vascular Endothelial Growth Factor; DME, Diabetic Macular Edema; PDR, Proliferative Diabetic Retinopathy; PRP, Panretinal photocoagulation; CMT, Central Macular Thickness; BCVA, Best Corrected Visual Acuity; DM, Diabetes Mellitus; NPDR, Non-Proliferative Diabetic Retinopathy; NV, Neovascularization; NVG, Neovascular Glaucoma; DRCR, Diabetic Retinopathy Clinical Research Network; ETDRS, Early Treatment Diabetic Retinopathy Study Research Group; FAG, Fluorescein Angiography; NVD, Neovascularization of the optic Disc; NVE, Neovascularization Elsewhere; IDDM, Insulin Dependent diabetes mellitus; NIDDM, Non Insulin Dependent diabetes mellitus; VMT, Vitreomacular Traction; PVR, Proliferative Vitreoretinopathy; SD-OCT, Spectral Domain Optical Coherence Tomography; HbA1C, Glycated Hemoglobin.

\section{Data Sharing Statement}

The datasets used and/or analysed during the current study are available from the corresponding author on reasonable request.

\section{Ethics Approval and Consent to Participate}

All authors certify that they have no affiliations with or involvement in any organization or entity with any financial interest (such as honoraria; educational grants; participation in speakers' bureaus; membership, employment, consultancies, stock ownership, or other equity interest; and expert testimony or patent-licensing arrangements), or non-financial interest (such as personal or professional relationships, affiliations, knowledge or beliefs) in the subject matter or materials discussed in this manuscript.

This study was approved by the Ethics Committee of the Medical Association of Saarland, Germany (Nr. 123/20). All procedures performed in studies involving human participants were in accordance with the ethical standards of the institutional and/or national research committee and with the 1964 Helsinki declaration and its later amendments or comparable ethical standards.

This article does not contain any studies with animals performed by any of the authors.

For this type of retrospective study, formal consent is not required

Patient and Public Involvement statement: Patients or the public were not involved in the design, or conduct, or reporting, or dissemination plans of our research

\section{Author Contributions}

All authors made a significant contribution to the work reported, whether that is in the conception, study design, execution, acquisition of data, analysis and interpretation, or in all these areas; took part in drafting, revising or critically reviewing the article; gave final approval of the version to be published; have agreed on the journal to which the article has been submitted; and agree to be accountable for all aspects of the work.

\section{Funding}

No funding was received for this research.

\section{Disclosure}

All authors certify that they have no affiliations with or involvement in any organization or entity with any financial interest (such as honoraria; educational grants; participation in speakers' bureaus; membership, employment, consultancies, stock ownership, or other equity interest; and expert testimony or patent-licensing arrangements), or non-financial 
interest (such as personal or professional relationships, affiliations, knowledge or beliefs) in the subject matter or materials discussed in this manuscript.

\section{References}

1. Hartnett ME, Baehr W, Le YZ. Diabetic retinopathy, an overview. Vision Res. 2017;139(11):1-6. doi:10.1016/j.visres.2017.07.006

2. Korobelnik JF, Do DV, Schmidt-Erfurth U, et al. Intravitreal aflibercept for diabetic macular edema. Ophthalmology. 2014;121(11):2247-2254. doi:10.1016/j.ophtha.2014.05.006

3. Cheung N, Wong IY, Wong TY. Ocular anti-VEGF therapy for diabetic retinopathy: overview of clinical efficacy and evolving applications. Diabetes Care. 2014;37(4):900-905. doi:10.2337/dc13-1990

4. Yau JWY, Rogers SL, Kawasaki R, et al. Global prevalence and major risk factors of diabetic retinopathy. Diabetes Care. 2012;35(3):556-564. doi:10.2337/dc11-1909

5. Shibuya M. Differential roles of vascular endothelial growth factor receptor-1 and receptor-2 in angiogenesis. BMB Rep. 2006;39(5):469-478. doi:10.5483/BMBRep.2006.39.5.469

6. Carmeliet P, Moons L, Luttun A, et al. Synergism between vascular endothelial growth factor and placental growth factor contributes to angiogenesis and plasma extravasation in pathological conditions. Nat Med. 2001;7(5):575-583. doi:10.1038/87904

7. Gillies MC, Lim LL, Campain A, et al. A randomized clinical trial of intravitreal bevacizumab versus intravitreal dexamethasone for diabetic macular edema: the BEVORDEX study. Ophthalmology. 2014;121(12):2473-2481. doi:10.1016/j.ophtha.2014.07.002

8. Joussen AM, Poulaki V, Qin W, et al. Retinal vascular endothelial growth factor induces intercellular adhesion molecule-1 and endothelial nitric oxide synthase expression and initiates early diabetic retinal leukocyte adhesion in vivo. Am J Pathol. 2002;160(2):501-509. doi:10.1016/S00029440(10)64869-9

9. Rungger-Brändle E, Dosso AA, Leuenberger PM. Glial reactivity, an early feature of diabetic retinopathy. Invest Ophthalmol Vis Sci. 2000;41 (7):1971-1980.

10. Zhang J, Gerhardinger C, Lorenzi M. Early complement activation and decreased levels of glycosylphosphatidylinositol-anchored complement inhibitors in human and experimental diabetic retinopathy. Diabetes. 2002;51(12):3499-34504. doi:10.2337/diabetes.51.12.3499

11. Antonetti DA, Barber AJ, Bronson SK, et al. Diabetic retinopathy: seeing beyond glucose-induced microvascular disease. Diabetes. 2006;55 (9):2401-2411. doi:10.2337/db05-1635

12. Cusick M, Chew EY, Chan -C-C, Kruth HS, Murphy RP, Ferris FL. Histopathology and regression of retinal hard exudates in diabetic retinopathy after reduction of elevated serum lipid levels. Ophthalmology. 2003;110(11):2126-2133. doi:10.1016/j.ophtha.2003.01.001

13. Sun JK, Glassman AR, Beaulieu WT, et al. Rationale and application of the protocol S anti-vascular endothelial growth factor algorithm for proliferative diabetic retinopathy. Ophthalmology. 2019;126(1):87-95. doi:10.1016/j.ophtha.2018.08.001

14. Early Treatment Diabetic Retinopathy Study Research Group. Fundus photographic risk factors for progression of diabetic retinopathy: ETDRS report number 12. Ophthalmology. 1991;98(5):823-833. doi:10.1016/S0161-6420(13)38014-2

15. Wang X, Wang G, Wang Y. Intravitreous vascular endothelial growth factor and hypoxia-inducible factor 1a in patients with proliferative diabetic retinopathy. Am J Ophthalmol. 2009;148(6):883-889. doi:10.1016/j.ajo.2009.07.007

16. Abdin AD, Suffo S, Bischoff-Jung M, Daas L, Pattmöller M, Seitz B. Advantages of a designated IVI center for a German university eye hospital. Der Ophthalmol Zeitschrift der Dtsch Ophthalmol Gesellschaft. 2020;117(1):50-57. doi:10.1007/s00347-019-0911-5

17. Lang GE, Stahl A, Voegeler J, et al. Efficacy and safety of ranibizumab with or without panretinal laser photocoagulation versus laser photocoagulation alone in proliferative diabetic retinopathy-the PRIDE study. Acta Ophthalmol. 2020;98(5):530-539. doi:10.1111/aos.14312

18. Sivaprasad S, Prevost AT, Vasconcelos JC, et al. Clinical efficacy of intravitreal aflibercept versus panretinal photocoagulation for best corrected visual acuity in patients with proliferative diabetic retinopathy at 52 weeks (CLARITY): a multicentre, single-blinded, randomised, controlled, phase 2b, non-inferiority trial. Lancet. 2017;389(10085):2193-2203. doi:10.1016/S0140-6736(17)31193-5

19. Ip MS, Domalpally A, Hopkins JJ, Wong P, Ehrlich JS. Long-term effects of ranibizumab on diabetic retinopathy severity and progression. Arch Ophthalmol. 2012;130(9):1145-1152. doi:10.1001/archophthalmol.2012.1043

20. Perretti M, Ahluwalia A. The microcirculation and inflammation: site of action for glucocorticoids. Microcirculation. $2000 ; 7(3): 147-161$. doi:10.1111/j.1549-8719.2000.tb00117.x

21. Herold MJ, McPherson KG, Reichardt HM. Glucocorticoids in T cell apoptosis and function. Cell Mol Life Sci. 2006;63(1):60-72. doi:10.1007/ s00018-005-5390-y

22. Coutinho AE, Chapman KE. The anti-inflammatory and immunosuppressive effects of glucocorticoids, recent developments and mechanistic insights. Mol Cell Endocrinol. 2011;335(1):2-13. doi:10.1016/j.mce.2010.04.005

23. Rübsam A, Parikh S, Fort PE. Role of inflammation in diabetic retinopathy. Int J Mol Sci. 2018;19(4):942. doi:10.3390/ijms19040942

24. Fallico M, Maugeri A, Lotery A, et al. Intravitreal anti-vascular endothelial growth factors, panretinal photocoagulation and combined treatment for proliferative diabetic retinopathy: a systematic review and network meta-analysis. Acta Ophthalmol. 2021;99(6):795-805. doi:10.1111/aos.14681

Clinical Ophthalmology

Dovepress

\section{Publish your work in this journal}

Clinical Ophthalmology is an international, peer-reviewed journal covering all subspecialties within ophthalmology. Key topics include: Optometry; Visual science; Pharmacology and drug therapy in eye diseases; Basic Sciences; Primary and Secondary eye care; Patient Safety and Quality of Care Improvements. This journal is indexed on PubMed Central and CAS, and is the official journal of The Society of Clinical Ophthalmology (SCO). The manuscript management system is completely online and includes a very quick and fair peer-review system, which is all easy to use. Visit http://www. dovepress.com/testimonials.php to read real quotes from published authors.

Submit your manuscript here: https://www.dovepress.com/clinical-ophthalmology-journal 